\title{
On the Problem of Characterizing Boolean Petri Nets
}

\author{
Sangita Kansal \\ Department of Applied Mathematics \\ Delhi Technological University \\ Shahbad Daulatpur, Main Bawana Road \\ Delhi-110042, India
}

\author{
Gajendra Pratap Singh \\ Department of Mathematics \\ Ramjas College \\ University of Delhi, Delhi \\ Delhi-110007, India
}

\author{
Mukti Acharya \\ (Visiting Professor) \\ Department of Mathematics \\ Kalasalingam University, Anand Nagar \\ Krishnankoil-626126, India
}

\begin{abstract}
Petri nets are used for describing, designing and studying discrete event-driven systems that are characterized as being concurrent, asynchronous, distributed, parallel, and/or nondeterministic. As a graphical tool, Petri net can be used for planning and designing a system with given objectives, more effectively than flowcharts and block diagrams. As a mathematical tool, it enables one to set up state equations and algebraic equations and other mathematical models which govern the behavior of systems. The aim of this paper is to present some basic results and necessary and sufficient condition for a 1-safe Petri net that generates all the binary $n$ vectors as marking vectors, we shall call such Petri nets as Boolean Petri nets.
\end{abstract}

\section{Keywords}

1-safe Petri net, reachability tree, binary $n$-vector, marking vector

\section{INTRODUCTION}

A Petri net is a graphical tool invented by Carl Adam Petri [9]. Petri nets are very reliable tool to model and study the structure of the discrete event-driven systems with large population or heavy traffic appear frequently in many fields such as manufacturing processes, logistics, telecommunication systems, traffic systems etc [8]. Of all existing models, Petri nets and their extensions are of undeniable fundamental interest because they define easy graphical support for the representation and the understanding of basic mechanism and behaviors. The development of high-end computers has greatly enhanced the use of Petri nets in diverse fields. Kansal et al.[5] showed the existence of 1-safe star Petri net $S_{n}$, with $|P|=n$ and $|T|=n+1$, having a central transition, that generates all the binary $n$-vectors, as its marking vectors; they also established the existence of 1-safe Petri net that generates all the binary $n$ vectors exactly once as marking vectors[6]. A 1-safe Petri net is called Boolean when it generates every binary $n$-vector as its marking vector and it is called Crisp Boolean when it generates every binary $n$-vector exactly once.

\section{PRELIMINARIES}

For standard terminology and notation on Petri nets, we refer the reader to Peterson [11]. In this paper, we shall adopt the definition of Jenson [3]:
A Petri net is a 5-tuple $N=\left(P, T, I^{-}, I^{+}, \mu^{0}\right)$, where

(a) $P$ is a nonempty set of 'places',

(b) $T$ is a nonempty set of 'transitions',

(c) $P \cap T=\emptyset$,

(d) $I^{-}, I^{+}: P \times T \longrightarrow \mathbb{N}$, where $\mathbb{N}$ is the set of nonnegative integers, are called the negative and the positive incidence functions' (or, 'flow functions') respectively,

(e) $\forall p \in P, \exists t \in T: I^{-}(p, t) \neq 0$ or $I^{+}(p, t) \neq 0$ and

$\forall t \in T, \exists p \in P: I^{-}(p, t) \neq 0$ or $I^{+}(p, t) \neq 0$,

(f) $\mu^{0}: P \rightarrow \mathbb{N}$ is the initial marking.

In fact, $I^{-}(p, t)$ and $I^{+}(p, t)$ represent the number of arcs from $p$ to $t$ and $t$ to $p$ respectively. $I^{-}, I^{+}$and $\mu^{0}$ can be viewed as matrices of size $|P| \times|T|,|P| \times|T|$ and $|P| \times 1$, respectively.

As in many standard books (e.g., see [12]), Petri net is a particular kind of directed graph, together with an initial marking $\mu^{0}$. The underlying graph of a Petri net is a directed, weighted, bipartite graph consisting of two kinds of nodes, called places and transitions, where arcs are either from a place to a transition or from a transition to a place. Hence, Petri nets have a well known graphical representation in which transitions are represented as boxes and places as circles with directed arcs interconnecting places and transitions to represent the flow relation. The initial marking is represented by placing a token in the circle representing a place $p_{i}$ as a black dot whenever $\mu^{0}\left(p_{i}\right)=1,1 \leq i \leq n=|P|$. In general, a marking $\mu$ is a mapping $\mu: P \longrightarrow \mathbb{N}$. A marking $\mu$ can hence be represented as a vector $\mu \in \mathbb{N}^{n}, n=|P|$, such that the $i^{\text {th }}$ component of $\mu$ is the value $\mu\left(p_{i}\right)$.

Let $N=\left(P, T, I^{-}, I^{+}, \mu\right)$ be a Petri net. A transition $t \in T$ is said to be enabled at $\mu$ if and only if $I^{-}(p, t) \leq \mu(p), \forall p \in P$. An enabled transition may or may not 'fire' (depending on whether or not the event actually takes place). After firing at $\mu$, the new marking $\mu^{\prime}$ is given by the rule

$$
\mu^{\prime}(p)=\mu(p)-I^{-}(p, t)+I^{+}(p, t), \text { for all } p \in P .
$$

A marking $\mu$ is said to be reachable from $\mu^{0}$, if there exists a sequence of transitions which can be successively fired to obtain $\mu$ from $\mu^{0}$. The set of all markings of a Petri net $N$ reachable from a given marking $\mu$ is denoted by $\mathcal{M}(N, \mu)$ and, together with the arcs of the form $\mu^{i} \stackrel{t_{r}}{\longrightarrow} \mu^{j}$, represents what in standard terminology called the reachability graph $R(N, \mu)$ of the Petri net $N$. If the reachability graph has no cycle then it is called 


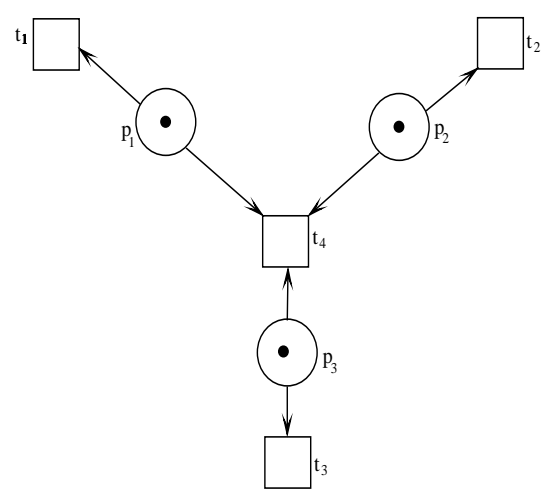

Fig. 1. Boolean Petri net with 3 places and 4 transitions satisfying $|P| \leq$ $|T|$

reachability tree of the Petri net $N$.

A place in a Petri net is 1-safe if the number of tokens in that place never exceeds one. A Petri net is 1-safe if all its places are 1 -safe. The preset of a transition $t$ is the set of all input places to $t$, i.e., ${ }^{\bullet} t=\left\{p \in P: I^{-}(p, t)>0\right\}$. The postset of $t$ is the set of all output places from $t$, i.e., $t^{\bullet}=\left\{p \in P: I^{+}(p, t)>0\right\}$. Similarly, $p^{\prime} s$ preset and postset are ${ }^{\bullet} p=\left\{t \in T: I^{+}(p, t)>0\right\}$ and $p^{\bullet}=\left\{t \in T: I^{-}(p, t)>0\right\}$, respectively.

Let $N=\left(P, T, I^{-}, I^{+}, \mu^{0}\right)$ be a Petri net with $|P|=n$ and $|T|=m$, the incidence matrix $I=\left[a_{i j}\right]$ is an $n \times m$ matrix of integers, $|P|=n$ and $|T|=m$ and its entries are given by $a_{i j}=a_{i j}^{+}-a_{i j}^{-}$where $a_{i j}^{+}=I^{+}\left(p_{i}, t_{j}\right)$ is the number of arcs from transition $t_{j}$ to its output place $p_{i}$, known as positive incidence matrix and $a_{i j}^{-}=I^{-}\left(p_{i}, t_{j}\right)$ is the number of arcs from place $p_{i}$ to its output transition $t_{j}$, known as negative incidence matrix. In other words, $I=I^{+}-I^{-}$.

\section{SOME GENERAL RESULTS}

In this section, we discuss some necessary conditions for a Boolean Petri net.

Proposition 1. 6] For any Boolean Petri net, $\mu^{0}(p)=$ $1, \forall p \in P$, where $\mu^{0}$ is the initial marking vector

Lemma 1. [4] If a 1-safe Petri net $N=\left(P, T, I^{-}, I^{+}, \mu^{0}\right)$, $|P|=n$, is Boolean then $|P| \leq|T|$.

Proof. Since $N$ is Boolean, it generates the marking vectors of the type $(0,1,1, \cdots, 1),(1,0,1, \cdots, 1), \cdots,(1,1,1, \cdots, 0)$, each having the Hamming distance 1 from the initial marking vector $\mu^{0}=(1,1,1, \cdots, 1)$. These $n$ marking vectors can be obtained only in the first step of firing because the marking vector whose Hamming distance is 1 from the initial marking cannot be obtained from any other marking vector whose Hamming distance is greater than or equal to 2 from the initial marking. These $n$ marking vectors can be generated only if for every place $p_{i} \in P, i=1,2,3, \cdots, n$, there exist distinct $n$ transitions say $t_{1}, t_{2}, t_{3}, \cdots, t_{n}$ such that $p_{i} \in$ $\cdot t_{i}$ and $p_{i} \notin t_{i}^{\bullet} \forall i=1,2,3, \cdots, n$. Hence, $|P| \leq|T|$.

The Figure 1 and Figure 2 are the explanation of lemma 1.

Lemma 2. [4] If a 1-safe Petri net $N=\left(P, T, I^{-}, I^{+}, \mu^{0}\right)$, $|P|=n$, is Boolean then the incidence matrix I of $N$ contains $-I_{n}$, the negative of the identity matrix of order $n$, as a submatrix.

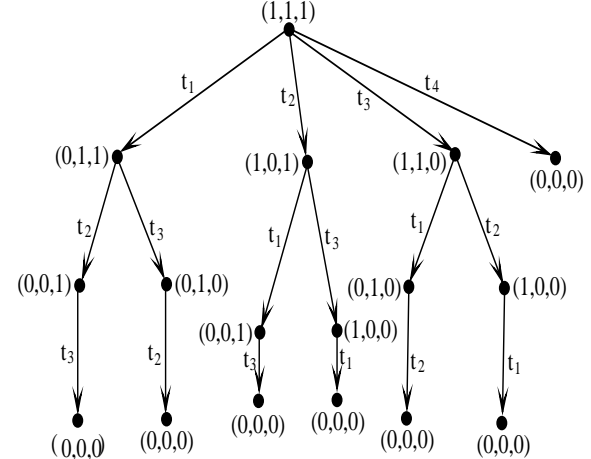

Fig. 2. Reachability tree of Figure 1 containing all the $2^{3}$ binary 3 -vectors

PROOF. Since $N$ generates all the binary $n$-vectors, $\mu^{0}(p)=$ 1, $\forall p \in P$ (by Proposition 1). Again, because of the generation of all the binary $n$-vectors, the vectors of the type $(0,1,1, \cdots, 1),(1,0,1, \cdots, 1), \cdots,(1,1,1, \cdots, 0)$ each at a Hamming distance 1 from the initial marking, have also been generated. These vectors can be obtained only in the first step of firing. Therefore, $\forall p_{i} \in P, i=1,2,3, \cdots, n$, there exist distinct $n$ transitions say $t_{1}, t_{2}, t_{3}, \cdots, t_{n}$ such that $p_{i} \in{ }^{\bullet} t_{i}$ and $p_{i} \notin t_{i}^{\bullet}$ and hence $I^{-}\left(p_{i}, t_{j}\right)=1$ if $i=j$ and 0 if $i \neq j$ and also $I^{+}\left(p_{i}, t_{i}\right)=0, \forall i=1,2,3, \cdots, n$. Since $I=I^{+}-I^{-}, I$ contains $-I_{n}$ as a submatrix.

The followings are the positive incidence matrix $\left(I^{+}\right)$, negative incidence matrix $\left(I^{-}\right)$and incidence matrix $I=I^{+}-I^{-}$respectively of the Figure 1 which contains $-I_{n}$ as a submatrix.

$$
\begin{aligned}
& I^{+}=\begin{array}{c}
p_{1} \\
p_{2} \\
p_{3}
\end{array}\left[\begin{array}{llll}
t_{1} & t_{2} & t_{3} & t_{4} \\
0 & 0 & 0 & 0 \\
0 & 0 & 0 & 0 \\
t_{1} & t_{2} & t_{3} & t_{4}
\end{array}\right] \\
& I^{-}=\begin{array}{l}
p_{1} \\
p_{2} \\
p_{3}
\end{array}\left[\begin{array}{llll}
1 & 0 & 0 & 1 \\
0 & 1 & 0 & 1 \\
0 & 0 & 1 & 1
\end{array}\right] \\
& I=I^{+}-I^{-}=\begin{array}{l}
p_{1} \\
p_{2} \\
p_{3}
\end{array}-\left[\begin{array}{llll}
1 & 0 & 0 & 1 \\
0 & 1 & 0 & 1 \\
0 & 0 & 1 & 1
\end{array}\right]
\end{aligned}
$$

\section{MAIN RESULT}

In this section, we will study necessary and sufficient conditions for a Boolean Petri nets as reported in [4].

Theorem 3. A 1-safe Petri net $N=\left(P, T, I^{-}, I^{+}, \mu^{0}\right)$, $|P|=n$ with $t^{\bullet}=\emptyset \forall t \in T$ is Boolean if and only if

1. $\mu^{0}(p)=1, \forall p \in P$

2. $|P| \leq|T|$

3. The incidence matrix I of $N$ contains $-I_{n}$ as a submatrix.

Proof. Necessity: This follows from Proposition 1, Lemma 1 and Lemma 2 respectively.

Sufficiency: Given the hypothesis and conditions (1), (2) and (3), we claim that $N$ generates all the binary $n$-vectors. Since $I=I^{+}-I^{-}$and $t^{\bullet}=\emptyset, \forall t \in T, I^{+}=0$. This implies that 
$I=-I^{-}$. Since $I$ contains $-I_{n}$ as a submatrix, $\forall p_{i} \in P, \exists t_{i} \in T$ such that $p_{i} \in{ }^{\prime} t_{i}, \forall i=1,2, \cdots, n$. Also, $\mu^{0}(p)=1, \forall p \in P$. Therefore, all the $n$ transitions $t_{1}, t_{2}, \cdots, t_{n}$ are enabled and fire. After firing, we get distinct ${ }^{n} C_{1}=n$ marking vectors whose Hamming distance is 1 from the initial marking vector. At these $n$ new marking vectors, $(n-1)$ transitions are enabled and give at least ${ }^{n} C_{2}$ distinct marking vectors, each of whose Hamming distance is 2 from the initial marking. Therefore, this set of new vectors contains at least ${ }^{n} C_{2}$ new distinct binary $n$-vectors.

In general at any stage $j, 3 \leq j \leq n$, we get a set of at least ${ }^{n} C_{j}$ new distinct binary $n$-vectors whose Hamming distance is $j$ from the initial marking, which are also distinct from the sets of ${ }^{n} C_{r}$ distinct marking vectors for all $r, 2 \leq r \leq j-1$. Therefore, at the $n^{\text {th }}$ stage we would have obtained at least ${ }^{n} C_{1}+{ }^{n}$ $C_{2}+\cdots+{ }^{n} C_{n}=2^{n}-1$ distinct binary $n$-vectors. Together with the initial marking $(1,1, \cdots, 1)$, we thus see that all the $2^{n}$ binary $n$-vectors would have been obtained as marking vectors, possibly with repetitions. Thus, $N$ is Boolean and the proof is complete.

Figure 1] Figure 2 and incidence matrix of Figure 1) clearly explain the above theorem.

Theorem 4. A 1-safe Petri net $N=\left(P, T, I^{-}, I^{+}, \mu^{0}\right)$, $|P|=n$, with $I^{-}\left(p_{i}, t_{j}\right)=1, \forall i, j$, is Boolean if and only if there exist at least ${ }^{n} C_{r}, r=1,2, \cdots, n$ distinct transitions $t \in T$ such that $\left|t^{\bullet}\right|=n-r$, where $r$ is the Hamming distance of any binary $n$-vector from the initial marking $(1,1, \cdots, 1)$.

PROOF. Necessity: Since $N$ generates all the binary $n$-vectors, we have the binary $n$-vectors $(0,1,1, \cdots, 1),(1,0,1, \cdots, 1)$, $(1,1,0, \cdots, 1), \cdots,(1,1,1, \cdots, 1,0)$, whose Hamming distance is 1 from the initial marking $(1,1,1, \cdots 1)$. They are $n$ in number. Since $I^{-}\left(p_{i}, t_{j}\right)=1, \forall i, j$, these vectors can be obtained only if $I^{+}\left(p_{i}, t_{j}\right)=0$ for $i=j$ and 1 for $i \neq j, 1 \leq j \leq n$. This implies that there are at least ${ }^{n} C_{1}$ distinct transitions say $t_{1}, t_{2}, \cdots, t_{n}$ such that $\left|t^{\bullet}\right|=n-1$. After firing, they become dead. Further, we also have the binary $n$-vectors $(0,0,1, \cdots, 1)$, $(1,0,0,1, \cdots, 1), \quad(1,0,1,0,1, \cdots, 1), \cdots,(1,1, \cdots, 1,0,0)$ each of whose Hamming distance is 2 from $(1,1,1, \cdots 1)$. These vectors are ${ }^{n} C_{2}$ in number and can be obtained only if there exist at least ${ }^{n} C_{2}$ distinct transitions $t$ with $\left|t^{\bullet}\right|=n-2$. In general, there are at least ${ }^{n} C_{r}$ distinct transitions $t$ such that $\left|t^{\bullet}\right|=n-r$, that yield ${ }^{n} C_{r}$ binary $n$-vectors each at Hamming distance $r$ from $(1,1,1, \cdots 1)$.

Sufficiency: Since $\mu^{0}(p)=1, \forall p$, all the transitions are enabled and fire. After firing they all become dead as $I^{-}\left(p_{i}, t_{j}\right)=1$, $\forall i, j$. This implies that the matrix $I^{-}$is of order $n \times m$ where $m>\left(2^{n}-1\right)$ and the matrix $I^{+}$gets constructed as follows. By hypothesis, there are at least ${ }^{n} C_{1}=n$ distinct transitions in $N$ say $t_{1}, t_{2}, \cdots, t_{n}$ which on firing generate all the binary $n$ vectors each having exactly one zero because $\left|t_{i}^{\bullet}\right|=n-1$ (without lose of generality, we assume that there is no $\operatorname{arc}$ from $t_{i}$ to $p_{i}$ i.e., $I^{+}\left(p_{i}, t_{i}\right)=0$ for $\left.i=1,2, \cdots, n\right)$. Thus, we place the transpose of these binary $n$-vectors as first $n$ columns in $I^{+}$matrix. Next, by hypothesis, we have ${ }^{n} C_{2}$ distinct transitions say $t_{n+1}, t_{n+2}, \cdots$, ${ }^{{ }^{n} C_{2}}$ such that $\left|t_{j}^{\bullet}\right|=n-2$. Since they all become dead after firing and $\left|t_{j}^{\bullet}\right|=n-2, n+1 \leq j \leq{ }^{n} C_{2}$ these must generate all the distinct binary $n$-vectors each having exactly two zeros. Hence, the transpose of these ${ }^{n} C_{2}$ vectors are placed as columns in the matrix $I^{+}$immediately after the previous $n={ }^{n} C_{1}$ columns. We are thus enabled by the hypothesis to construct successively the submatrix $H$ of order $n \times\left(2^{n}-1\right)$ of $I^{+}$which contains all the $\left(2^{n}-1\right)$

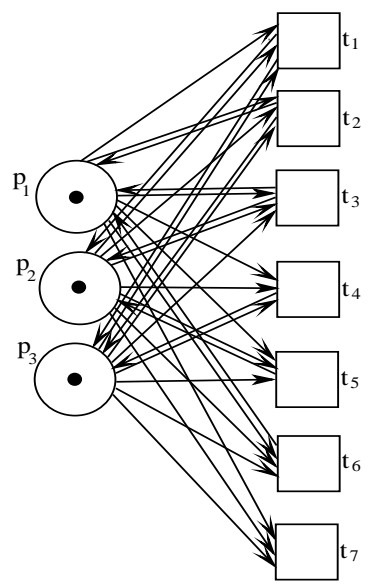

Fig. 3. Petri net with $I^{-}\left(p_{i}, t_{j}\right)=1$ and atleast ${ }^{n} C_{r}, r=1,2, \cdots, n$ transitions such that $\left|t^{\bullet}\right|=n-r$

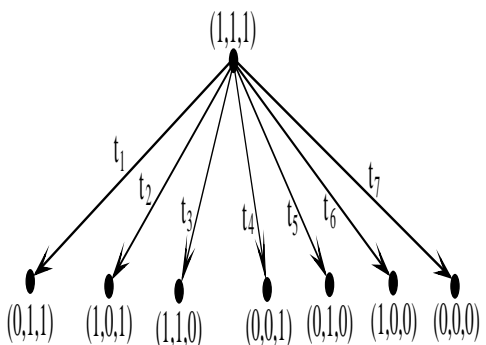

Fig. 4. Reachability tree of Figure 3 containing all the $2^{3}$ binary 3 -vectors

distinct binary $n$-vectors, the last column of $H$ being the all zero $n$-vector. We may augment to $H$ the initial all-one $n$-vector as a column either on the extreme left or on the extreme right of $H$ in $I^{+}$. Let the so augmented submatrix of $I^{+}$have more columns. That means, each one of them is a repetition of some columns in $H$. Thus, we see that the Petri net $N$ generates all the binary $n$-vectors as its marking vectors. Hence $N$ is a Boolean Petri net.

See Figure 3 and Figure 4 for the explanation of Theorem 4.

\section{CONCLUSIONS AND SCOPE}

As a conclusion, one can ask for the criteria that a 1-safe Petri net should satisfy for its reachability tree to be finite [13]. While solution to such a problem can perhaps be used gainfully in many purely theoretical areas like mathematics, computer science, universal algebra and order theory, decision theory|2], the extent and effectiveness of its utility in solving the practical problem requiring the design of multi-functional switches for the operation of certain discrete dynamical systems of common use such as washing machines and teleprinters (e.g., see [1, 7, 10]) can be explored instantly. So, the characterization of Boolean Petri nets is an open problem. Also, it appears that a computationally good characterization of Boolean Petri nets is still distant. In general, the characterization of Boolean Petri nets is an open problem. 


\section{REFERENCES}

[1] Acharya, B.D. 2001. Set-indexers of a graph and set-graceful graphs, Bull. Allahabad Math. Soc., India, 16, 1-23.

[2] Araki, T., Kasami, T. 1977. Some Decision Problems Related to the Reachability Problem for Petri Nets, Theoretical Computer Science 3(1), 85104

[3] Jensen, K. 1986. Coloured Petri nets, Lecture notes in Computer Science, Vol. 254, Springer-Verlag, Berlin, 248-299.

[4] Kansal, S., Acharya, M. and Singh, G.P. 2012. Boolean Petri nets. In: Petri nets - Manufacturing and Computer Science(Ed.: Pawel Pawlewski), 381-406; Chapter 17. In-Tech Global Publisher, ISBN 978-953-51-0700-2.

[5] Kansal, S., Singh, G.P., Acharya, M. 2010. On Petri nets generating all the binary n-vectors, Scientiae Mathematicae Japonicae, 71(2), 209-216:e-2010, 113-120.

[6] Kansal, S., Singh, G.P., Acharya, M. 2011. 1-Safe Petri nets generating every binary n-vector exactly once, Scientiae Mathematicae Japonicae, 74,(1), 29-36, e-2011, 127-134.

[7] Narendra, K.S., Balakrishnan, J. 1994. Adaptive control using multiple models and switching. Technical report, Yale University.

[8] Nawel, G., Claude, D., Malika, I. 2009. Colored stochastic Petri nets for modelling and analysis of multiclass retrial systems, Elsevier, 49(7-8), 14361448.

[9] Petri, C.A., 1962. Kommunikation mit automaten, Schriften des Institutes fur Instrumentelle Mathematik, Bonn.

[10] Pait, F.M, Morse, A.S. 1994. A cyclic switching strategy for parameter-adaptive control. IEEE Transactions on Automatic Control, 39(6), 11721183.

[11] Peterson, J. L., 1981. Petri net Theory and the Modeling of Systems, Englewood Cliffs, NJ: Prentice-Hall, Inc.

[12] Reisig,W., 1985. Petri nets, Springer-Verleg, New York, 1985.

[13] Tomihisa, K., Satoru, Kawai. 1989. An algorithm for drawing general undirected graphs, Information Processing Letters, Elsevier, 31(1), 7-15.

\footnotetext{
${ }^{1}$ The results of this paper has been appeared in Boolean Petri nets. In: Petri nets - Manufacturing and Computer Science(Ed.: Pawel Pawlewski), 381406; Chapter 17. In-Tech Global Publisher, 2012, ISBN 978-953-51-07002.
} 